\title{
Existência e multiplicidade de soluções para uma equação elítica quaselinear do tipo Kirchhoff
}

\author{
Existence and multiplicity of solutions for a quasilinear elliptic equation of \\ Kirchhoff type
}

\author{
Francisco Helmuth Soares Dias ${ }^{1}$ e Márcio Luís Miotto ${ }^{2}$ \\ ${ }^{1}$ Universidade Federal do Rio Grande do Sul, RS, Brasil \\ kxchico@yahoo.com.br \\ ${ }^{2}$ Universidade Federal de Santa Maria, RS, Brasil \\ miottomatica@gmail.com
}

\section{Resumo}

O objetivo deste trabalho é apresentar condições suficientes para a existência e multiplicidade de soluções da seguinte classe de problemas do tipo Kirchhoff:

$$
\left\{\begin{array}{l}
-\left(a+b\|u\|^{p}\right)^{p-1} \Delta_{p} u=h(x)|u|^{q}+f(x, u), x \in \Omega, \\
0 \lessgtr u \in W_{0}^{1, p}(\Omega),
\end{array}\right.
$$

onde $a, b>0$ são constantes, $\Delta_{p} u=\operatorname{div}\left(|\nabla u|^{p-2} \nabla u\right)$ é o operador $p$-Laplaciano com $p>1$, $\Omega$ é um domínio limitado suave em $\mathbb{R}^{N}$, onde $1<q+1<p<N<\frac{p^{2}}{p-1}$ e as funções $h(x)$ e $f(x, s)$ satisfazem condições apropriadas. Utilizaremos neste propósito argumentos variacionais, tais como o Teorema do Passo da Montanha e o Princípio Variacional de Ekeland.

Palavras-chave: Equação de Kirchhoff, p-Laplaciano, métodos variacionais, multiplicidade de soluções.

\section{Resumo}

The aim of this work is to give some sufficient conditions for the existence and multiplicity of the solutions for the following class of Kirchhoff type problems:

$$
\left\{\begin{array}{l}
-\left(a+b\|u\|^{p}\right)^{p-1} \Delta_{p} u=h(x)|u|^{q}+f(x, u), x \in \Omega, \\
0 \lesseqgtr u \in W_{0}^{1, p}(\Omega),
\end{array}\right.
$$

where $a, b>0$ are constants, $\Delta_{p} u=\operatorname{div}\left(|\nabla u|^{p-2} \nabla u\right)$ is the $p$-Laplacian operator with $p>1, \Omega$ is a bounded smooth domain in $\mathbb{R}^{N}$, with $1<q+1<p<N<\frac{p^{2}}{p-1}$ and the functions $h(x)$ and $f(x, s)$ satisfy appropriate conditions. We will use for this purpose variational arguments, such as Mountain Pass Theorem and the Ekeland Variational Principle. Keywords: Kirchhoff Equation, $p$-Laplacian, variational methods, multiplicity of solutions. 


\section{Introdução}

O problema clássico de Kirchhoff

$$
\left\{\begin{aligned}
-M\|u\|^{2} \Delta u & =f(x, u), x \in \Omega, \\
u & =0, x \in \partial \Omega
\end{aligned}\right.
$$

onde $M:[0,+\infty) \longrightarrow[0,+\infty)$ é uma função contínua, é a versão estacionária da equação

$$
\rho \frac{\partial^{2} u}{\partial t^{2}}-\left(\frac{P_{0}}{h}+\frac{E}{2 L} \int_{0}^{L}\left|\frac{\partial u}{\partial x}\right|^{2} d x\right) \frac{\partial^{2} u}{\partial x^{2}}=0,
$$

proposta por Kirchhoff (1883), a qual é uma generalização da conhecida equação de corda vibrante de D’Alembert. O modelo descrito em (2) leva em conta as mudanças no comprimento da corda produzida por vibrações transversais, sendo $L$ o comprimento da corda, $h$ a área da seção transversal, $E$ o módulo de Young do material, $\rho$ a densidade da massa e $P_{0}$ a tensão inicial. Relacionado com (1), Perera e Zhang (2006) abordaram o problema:

$$
\left\{\begin{aligned}
-\left(a+b\|u\|^{2}\right) \Delta u & =g(x, u), x \in \Omega, \\
u & =0, x \in \partial \Omega,
\end{aligned}\right.
$$

onde $\Omega \subset \mathbb{R}^{N}$ é um domínio limitado suave, $N \geq 1$ e $a, b>0$. A função $g(x, t)$ é localmente Lipschitz contínua em $t \in \mathbb{R}$, uniformemente contínua em $x \in \bar{\Omega}$ e subcrítica: $|g(x, t)| \leq C\left(|t|^{p-1}+1\right)$, para algum $2<p<2^{*}$, onde $C$ é uma constante positiva. Eles obtiveram via métodos variacionais a existência de uma solução positiva, uma solução negativa e uma solução mudando de sinal para o problema (3).

Por sua vez, Liang et al. (2013) via o argumento do grau topológico e métodos variacionais obtiveram a existência de soluções positivas para o problema:

$$
\left\{\begin{aligned}
-\left(a+b\|u\|^{2}\right) \Delta u & =\tau f(x, u), x \in \Omega, \\
u & =0, x \in \partial \Omega,
\end{aligned}\right.
$$

onde $\Omega \subset \mathbb{R}^{N}$ é um domínio limitado suave, $N=1,2$ ou $3, a, b \geq 0$, com $a+b>0$, $\tau$ é um parâmetro positivo e $f(x, t)$ é uma função contínua que é assintoticamente linear em zero e assintoticamente 3-linear no infinito, com relação a $t$.

Destacamos também, o importante trabalho de Li et al. (2002), que através do Princípio Variacional de Ekeland e do Teorema do Passo da Montanha, provaram a existência e multiplicidade de soluções positivas para a seguinte classe de problemas elíticos:

$$
\left\{\begin{aligned}
-\Delta u & =h(x) u^{q}+f(x, u), \quad x \in \Omega, \\
0 & \leq u \in W_{0}^{1, p}(\Omega),
\end{aligned}\right.
$$

onde $\Omega \subset \mathbb{R}^{N}$ é um domínio limitado suave, $N \geq 1,0<q<1, h \in L^{\infty}(\Omega), h(x) \not \equiv 0$, e a função $f(x, s)$ contempla os casos de crescimento assintoticamente linear e superlinear com relação a variável $s$ no infinito e o caso linear em $s$.

Mencionamos ainda o trabalho de Corrêa e Figueiredo (2006) o qual obteve resultados de existência de soluções para o problema

$$
\left\{\begin{aligned}
-\left[M\left(\|u\|^{p}\right)\right]^{p-1} \Delta_{p} u & =f(x, u)+\lambda|u|^{s-2} u, \quad x \in \Omega, \\
u & =0, x \in \partial \Omega,
\end{aligned}\right.
$$

onde $\Omega \subset \mathbb{R}^{N}$ é um domínio limitado suave, $1<p<N$, s $\geq p^{*}, \lambda \geq 0$, e $M$ e $f(x, t)$ satisfazem certas propriedades. Recentemente Guo e Nie (2015) obtiveram, sob certas condições, resultados de existência de infinitas soluções para a seguinte equação

$$
\left\{\begin{array}{c}
-\left(a+b\|u\|^{p}\right)^{p-1} \Delta_{p} u+\lambda V(x)|u|^{p-2} u=f(x, u) \\
u \in W^{1, p}\left(\mathbb{R}^{N}\right)
\end{array}\right.
$$

onde $p \geq 2, N<\frac{p^{2}}{p-1}, a>0, b \geq 0$ são constantes, $\lambda$ é um parâmetro e $V(x)$ é uma função potencial.

Citamos ainda dentre outros, os trabalhos de Alves et al. (2005), Dai (2009), Dai e Liu (2009), Dai e Wei (2010), Alves et al. (2010), Miotto (2010), Cammaroto e Vilasi (2011), Colasuonno e Pucci (2011),Sun e Tang (2011), Pei (2012), Huang et al. (2013), Hssini et al. (2013), Ferrara et al. (2014), Miotto (2014) e Hssini et al. (2015) os quais utilizam argumentos variacionais.

No presente trabalho, fazendo o uso de métodos variacionais, apresentamos resultados de existência e multiplicidade de soluções fracas não negativas da seguinte classe de problemas do tipo Kirchhoff: 


$$
\left\{\begin{array}{l}
-\left(a+b\|u\|^{p}\right)^{p-1} \Delta_{p} u=h(x)|u|^{q}+f(x, u), \\
0 \lesseqgtr u \in W_{0}^{1, p}(\Omega)
\end{array}\right.
$$

onde $a, b>0$ são constantes, $\Delta_{p} u=\operatorname{div}\left(|\nabla u|^{p-2} \nabla u\right)$ é o operador $p$-Laplaciano com $p>1, \Omega$ é um domínio limitado suave em $\mathbb{R}^{N}$, com os expoentes $1<q+1<p, p<N<\frac{p^{2}}{p-1}$ e as funções $h(x)$ e $f(x, s)$ satisfazem as seguintes hipóteses:

(h1) $h \in L^{\infty}(\Omega)$ e $h(x)>0$ em $\Omega_{0} \subset \Omega$;

(f1) $f(x, s) \in C(\bar{\Omega} \times[0, \infty)), f(x, 0)=0$;

(f2) $\lim _{s \rightarrow 0^{+}} \frac{f(x, s)}{\lambda_{1}(a s)^{p-1}}=\alpha, \lim _{s \rightarrow \infty} \frac{f(x, s)}{\mu_{1} b^{p-1} s^{p^{2}-1}}=\beta$, q.t.p. $x \in \Omega \operatorname{com} 0 \leq \alpha<1<\beta<\infty \mathrm{e}$

$$
\lambda_{1}=\inf \left\{\frac{\|u\|^{p}}{\|u\|_{p}^{p}} ; u \in W_{0}^{1, p}(\Omega), u \neq \equiv\right\}>0
$$

Sob tais condições obtemos o seguinte resultado de existência:

Teorema 1.1. Suponhamos que as condições $(h 1),(f 1)$ e $(f 2)$ sejam válidas. Então existe uma constante $\Lambda>0$ tal que se $\|h\|_{\infty}<\Lambda$, o problema (8) possui ao menos uma solução $u_{1} \in W_{0}^{1, p}(\Omega)$, com $u_{1} \gtrless 0$. Além disso, se $h(x) \geq 0$, então $u_{1}$ é positiva q.t.p. em $\Omega$.

Além disso, se supormos que é válida a seguinte condição:

(f3) Existem $C, r>0$ de modo que

$$
p^{2} F(x, s)-f(x, s) s \leq C s^{p}
$$

q.t.p. $x \in \Omega$ se $s \geq r$, onde $F(x, s)=\int_{0}^{s} f(x, t) d t$.

Então obtemos o seguinte resultado a respeito da multiplicidade de soluções de (8):

Teorema 1.2. Suponhamos válidas as condições $(h 1),(f 1),(f 2),(f 3)$ com $\|h\|_{\infty}<\Lambda$. Então o problema (8) admite uma segunda solução não negativa $u_{2} \in W_{0}^{1, p}(\Omega)$. Além disso, $u_{2}>0$ q.t.p. em $\Omega$, se $h(x) \geq 0$.

Este artigo está organizado da seguinte forma: na primeira seção, exploramos alguns resultados preliminares para este trabalho. Na seção seguinte, através do Princípio Variacional de Ekeland justificamos o Teorema 1.1. Ainda na referida seção, por meio do Teorema do Passo da Montanha faremos a demostração do Teorema 1.2.

\section{Resultados Preliminares}

Iniciamos essa seção apresentando algumas convenções. Temos que o expoente crítico de Sobolev é dado por $p^{*}=\frac{N p}{N-p}$. Como de costume, denotaremos a norma do espaço de Lebesgue $L^{r}(\Omega)$ por

$$
\begin{gathered}
\|u\|_{r}^{r} \doteq \int_{\Omega}|u|^{r} d x, \text { se } 1 \leq r<\infty, \\
\|u\|_{\infty} \doteq \text { ess } \sup _{\Omega}|u| .
\end{gathered}
$$

Definimos o espaço de Sobolev $W_{0}^{1, p}(\Omega)$ como sendo o fecho de $C_{c}^{\infty}(\Omega)$ em $W^{1, p}(\Omega)$, com respeito a norma

$$
\|u\|=\left(\int_{\Omega}|\nabla u|^{p} d x\right)^{1 / p}
$$

Dizemos que $u \in W_{0}^{1, p}(\Omega)$ é uma solução fraca para o problema (8), se $u$ satisfaz a equação

$$
\left(a+b\|u\|^{p}\right)^{p-1} \int_{\Omega}|\nabla u|^{p-2} \nabla u \nabla \varphi d x=\int_{\Omega} h(x)|u|^{q-1} u \varphi d x+\int_{\Omega} f\left(x, u_{+}\right) \varphi d x
$$

para toda função $\varphi \in C_{c}^{\infty}(\Omega)$, onde $u_{+}=\max \{0, u\}$.

A ideia principal dos métodos variacionais é relacionar a existência de solução de uma equação à existência de um ponto crítico de um funcional associado a equação. Associamos então, ao problema (8) o funcional $I: W_{0}^{1, p}(\Omega) \longrightarrow \mathbb{R}$, definido por 


$$
I(u)=\frac{1}{b p^{2}}\left(a+b\|u\|^{p}\right)^{p}-\frac{1}{q+1} \int_{\Omega} h(x)|u|^{q+1} d x-\int_{\Omega} F\left(x, u_{+}\right) d x
$$

onde $F(x, t)=\int_{0}^{t} f(x, s) d s$.

Através das hipóteses $(h 1),(f 1)$ e $(f 2)$, mostra-se que $I \in C^{1}\left(W_{0}^{1, p}(\Omega), \mathbb{R}\right)$ e apresenta derivada $I^{\prime}(u)$ em cada $u \in W_{0}^{1, p}(\Omega)$ dada por

$$
\left\langle I^{\prime}(u), \varphi\right\rangle=\left(a+b\|u\|^{p}\right)^{p-1} \int_{\Omega}|\nabla u|^{p-2} \nabla u \nabla \varphi d x-\int_{\Omega} h(x)|u|^{q-1} u \varphi d x-\int_{\Omega} f\left(x, u_{+}\right) \varphi d x,
$$

para cada $\varphi \in C_{c}^{\infty}(\Omega)$. Como por definição temos que $u \in W_{0}^{1, p}(\Omega)$ é um ponto crítico do funcional $I$ caso $I^{\prime}(u)=0$ em $\left(W_{0}^{1, p}(\Omega)\right)^{*}$, temos que os pontos críticos de $I$ são soluções fracas de $(8)$.

Abaixo, citamos alguns fatos relevantes para o estudo do problema (8).

Observação 2.1. (i) Consideremos $c \in \mathbb{R}$. Uma sequência $\left(u_{n}\right) \subset W_{0}^{1, p}(\Omega)$ tal que $I\left(u_{n}\right)=c+o(1)$ e $I^{\prime}\left(u_{n}\right)=o(1)$ em $W_{0}^{-1, p^{\prime}}(\Omega)$ é dita ser uma sequência $(P S)_{c}$ para o funcional I. Se qualquer sequência $(P S)_{c}$ para o funcional I, possui uma subsequência convergente, então dizemos que I satisfaz a condição (PS) de nível c.

(ii) Se $\left(u_{n}\right)$ é uma sequência $(P S)_{c}$ limitada para o funcional I, então $\left(u_{n_{+}}\right)$também é sequência $(P S)_{c}$ para o funcional I. Com efeito, sendo $\left(u_{n}\right)$ uma sequência $(P S)_{c}$ para o funcional $I$, então para cada $\varphi \in C_{c}^{\infty}(\Omega)$

$$
\left\langle I^{\prime}\left(u_{n}\right), \varphi\right\rangle=o(1),
$$

Tomando $\varphi=u_{n_{-}}$na igualdade acima, decorre que

$$
\left(a+b\left\|u_{n}\right\|^{p}\right)^{p}\left\|u_{n_{-}}\right\|^{p}-\int_{\Omega} f\left(x, u_{n_{+}}\right) u_{n_{-}} d x=o(1)
$$

e usando o fato de que $\int_{\Omega} f\left(x, u_{n_{+}}\right) u_{n_{-}} d x=0$, obtemos que

$$
\left\|u_{n_{-}}\right\|=o(1)
$$

$\log 0$

$$
\begin{aligned}
I\left(u_{n}\right)-I\left(u_{n_{+}}\right) & =\frac{1}{b p^{2}}\left[\left(a+\left\|u_{n}\right\|^{p}\right)^{p}-\left(a+\left\|u_{n_{+}}\right\|^{p}\right)^{p}\right. \\
& =o(1) .
\end{aligned}
$$

Por sua vez, como $\left(u_{n}\right)$ é limitada e $\left\|u_{n}\right\|=\left\|u_{n_{+}}\right\|+o(1)$, temos toda $\varphi \in C_{c}^{\infty}(\Omega)$

$$
\begin{aligned}
& <I^{\prime}\left(u_{n}\right)-I^{\prime}\left(u_{n_{+}}\right), \varphi> \\
= & {\left[\left(a+b\left\|u_{n}\right\|^{p}\right)^{p-1}-\left(a+b\left\|u_{n_{+}}\right\|^{p}\right)^{p-1}\right] } \\
& \times \int_{\Omega}\left|\nabla u_{n_{+}}\right|^{p-2} \nabla u_{n_{+}} \nabla \varphi d x+\left(a+b\left\|u_{n}\right\|^{p}\right)^{p-1} \int_{\Omega}\left|\nabla u_{n_{-}}\right|^{p-2} \nabla u_{n_{-}} \nabla \varphi d x \\
= & o(1),
\end{aligned}
$$

donde segue que $\left(u_{n_{+}}\right)$também é uma sequência $(P S)_{c}$ para o funcional I.

A seguir, aprentamos uma condição para a validade da condição (PS) em um certo nível $c$.

Lema 2.1. Suponhamos válidas as condições $(h 1),(f 1)$ e $(f 2)$ e que $\left(u_{n}\right) \subset W_{0}^{1, p}(\Omega)$ é uma sequência $(P S)_{c}$ limitada para o funcional I. Então $\left(u_{n}\right)$ possui uma subsequência convergente em $W_{0}^{1, p}(\Omega)$.

Demonstração: Seja $\left(u_{n}\right)$ uma sequencia $(P S)_{c}$ limitada para o funcional $I$, com $u_{n} \geq 0$. Então, passando a uma subsequência se necessário, podemos supor que

$$
\left\|u_{n}\right\|^{p} \rightarrow c_{0}
$$


e que existe $0 \leq u \in W_{0}^{1, p}(\Omega)$, de modo que $u_{n} \rightarrow u$ em $W_{0}^{1, p}(\Omega)$. Pelas condições $(h 1)$, $(f 1)$ e $(f 2)$, as imersões de Sobolev e o Teorema da Convergência Dominada, segue que

$$
\begin{aligned}
\int_{\Omega} f\left(x, u_{n}\right) u d x & =\int_{\Omega} f(x, u) u d x+o(1), \\
\int_{\Omega} f\left(x, u_{n}\right) u_{n} d x & =\int_{\Omega} f(x, u) u d x+o(1), \\
\int_{\Omega} h(x)\left|u_{n}\right|^{q-1} u_{n} u d x & =\int_{\Omega} h(x)\left|u_{n}\right|^{q+1} d x+o(1) \\
& =\int_{\Omega} h(x)|u|^{q+1} d x+o(1) .
\end{aligned}
$$

Assim, se considerarmos

$$
\begin{aligned}
c_{n}= & <I^{\prime}\left(u_{n}\right), u_{n}>+\int_{\Omega} h(x)\left|u_{n}\right|^{q+1} d x+\int_{\Omega} f\left(x, u_{n}\right) u d x-<I^{\prime}\left(u_{n}\right), u> \\
& -\int_{\Omega} h(x)\left|u_{n}\right|^{q-1} u_{n} u d x-\int_{\Omega} f\left(x, u_{n}\right) u d x,
\end{aligned}
$$

segue das relações $(9),(10),(11)$ e do fato que $\left(u_{n}\right)$ é sequencia $(P S)_{c}$ para o funcional $I$, que $c_{n}=o(1)$. Por outro lado,

$$
c_{n}=\left(a+b\left\|u_{n}\right\|^{p}\right)^{p-1} \int_{\Omega}\left|\nabla u_{n}\right|^{p-2} \nabla u_{n}\left(\nabla u_{n}-\nabla u\right) d x
$$

Agora pelo fato que $\left\|u_{n}\right\|^{p} \rightarrow c_{0}$ e $u_{n} \rightarrow u$ em $W_{0}^{1, p}(\Omega)$, segue que

$$
\left(a+b\left\|u_{n}\right\|^{p}\right)^{p-1} \int_{\Omega}|\nabla u|^{p-2} \nabla u\left(\nabla u_{n}-\nabla u\right) d x=o(1) .
$$

Assim, pelas relações acima, sendo $\ll \cdot, \gg$ o produto interno euclidiano em $\mathbb{R}^{N}$, temos que

$$
\left(a+b\left\|u_{n}\right\|^{p}\right)^{p-1} \int_{\Omega} \ll\left|\nabla u_{n}\right|^{p-2} \nabla u_{n}-|\nabla u|^{p-2} \nabla u, \nabla u_{n}-\nabla u \gg d x=o(1) .
$$

Agora usando o fato que existe $C=C(p)>0$ de modo que para todo $x, y \in \mathbb{R}^{N}$, temos se $p \geq 2$ ou $1<p<2$ respectivamente que,

$$
\begin{gathered}
\ll|x|^{p-2} x-|y|^{p-2} y, y-y \gg \geq C|x-y|^{p}, \\
\ll|x|^{p-2} x-|y|^{p-2} y, y-y \gg \geq C \frac{|x-y|^{2}}{(|x|+|y|)^{2-p}},
\end{gathered}
$$

obtemos por $\left\|u_{n}\right\|^{p} \rightarrow c_{0}$ e a relação (12) que existe $C>0$ onde

$$
\left(a+b c_{0}^{p}\right)^{p-1} C\left\|u_{n}-u\right\|^{p}=o(1),
$$

donde segue que $u_{n} \rightarrow u$ em $W_{0}^{1, p}(\Omega)$, o que finaliza a justificativa.

Enunciamos agora dois resultados clássicos da teoria dos pontos críticos, a saber, o Princípio Variacional de Ekeland e o Teorema do Passo da Montanha. Começamos pelo Princípio Variacional de Ekeland, cuja demonstração pode ser encontrada em de Figueiredo (1989).

Teorema 2.1 (Princípio Variacional de Ekeland). Seja $(X, d)$ um espaço métrico completo e $\phi: X \longrightarrow \mathbb{R} \cup\{\infty\}$ um funcional de classe $C^{1}$ e limitado inferiormente. Então se

$$
c=\inf _{u \in X} \phi(u)<\inf _{u \in \partial X} \phi(u),
$$

existe uma sequência $\left(u_{n}\right)$ em X satisfazendo a condição $(P S)_{c}$. 
O próximo resultado é o Teorema do Passo da Montanha que pode ser encontrado em Schechter (1991).

Teorema 2.2 (Teorema do Passo da Montanha). Seja X um espaço de Banach real e $\phi: X \longrightarrow \mathbb{R}$ um funcional de classe $C^{1}$ satisfazendo:

$$
\max \{\phi(0), \phi(v)\} \leq \kappa<v \leq \inf _{\|u\|=r} \phi(u)
$$

para algum $r>0$ e $v \in X$ com $\|v\|>r$. Então, existe uma sequência $\left(u_{n}\right)$ em $X$ satisfazendo a condição $(P S)_{c}$, onde $c \geq v$ pode ser caracterizado por

$$
c=\inf _{\sigma \in \Gamma} \max _{t \in[0,1]} \phi(\sigma(t))
$$

onde

$$
\Gamma=\{\sigma \in C([0,1], X) ; \sigma(0)=0, \sigma(1)=v\}
$$

Com o objetivo de obter sequências $(P S)_{c}$ para o funcional $I$ fazendo o uso dos resultados acima mencionados, vamos a seguir obter alguns resultados auxiliares sobre as funções $f(x, s)$ e $F(x, s)$ baseados nas condições $(f 1)$ e $(f 2)$. Pela hipótese $(f 2)$, temos que dado $\varepsilon \in(0,1)$, existe $\delta>0$ tal que para $s \in \mathbb{R} \cap(0, \delta)$ vale

$$
\left|\frac{f(x, s)}{\lambda_{1}(a s)^{p-1}}-\alpha\right|<\varepsilon .
$$

Logo, para $0<s<\delta$, temos

$$
(\alpha-\varepsilon) \lambda_{1}(a s)^{p-1}<f(x, s)<(\alpha+\varepsilon) \lambda_{1}(a s)^{p-1} .
$$

Por outro lado, de $\lim _{s \rightarrow \infty} \frac{f(x, s)}{b^{p-1} \mu_{1} s^{p^{2}-1}}=\beta$, temos que existe $\delta_{1}>\delta$, de modo que $0<\delta_{1}<s$, obtemos

$$
\left|\frac{f(x, s)}{b^{p-1} \mu_{1} s p^{2}-1}-\beta\right|<\varepsilon,
$$

e por conseguinte

$$
(\beta-\varepsilon) b^{p-1} \mu_{1} s^{p^{2}-1}<f(x, s)<(\beta+\varepsilon) b^{p-1} \mu_{1} s^{p^{2}-1} .
$$

Das desigualdades (13), (14) e como $f \in C(\bar{\Omega} \times[0, \infty))$, existe $C_{\varepsilon}>0$ de modo que para todo $s \in[0, \infty)$

$$
-C_{\varepsilon} s^{p-1}+(\beta-\varepsilon) b^{p-1} \mu_{1} s^{p^{2}-1}<f(x, s)<(\alpha+\varepsilon) \lambda_{1}(a s)^{p-1}+C_{\varepsilon} s p^{p^{2}-1} .
$$

Integrando em $s$ a expressão acima, obtemos para $s \geq 0$,

$$
-\frac{C_{\varepsilon}}{p} s^{p}+\frac{1}{p^{2}}(\beta-\varepsilon) b^{p-1} \mu_{1} s^{p^{2}}<F(x, s)<\frac{1}{p}(\alpha+\varepsilon) a^{p-1} \lambda_{1} s^{p}+\frac{C_{\varepsilon}}{p^{2}} s^{p^{2}} .
$$

O próximo resultado será essencial para garantirmos as hipóteses do Teorema do Passo da Montanha, bem como para a demonstração da existência de uma solução através do Princípio Variacional de Ekeland.

Lema 2.2. Suponhamos que as condições $(h 1),(f 1) e(f 2)$ sejam satisfeitas. Então existem $r, \gamma>0 e \Lambda>0, \Lambda=$ $\Lambda(a, \alpha, p, q, f, N, \Omega)$, tal que para qualquer $h \in L^{\infty}(\Omega)$ com $\|h\|_{\infty}<\Lambda$ temos que

$$
I(u) \geq I(0)+\gamma,
$$

para todo $u \in W_{0}^{1, p}(\Omega)$, com $\|u\|=r$.

Além disso, existe $v_{0} \in W_{0}^{1, p}(\Omega)$ com $\left\|v_{0}\right\|>r$, tal que $I\left(v_{0}\right)<I(0)$.

Demonstração: Utilizando o fato que para todo $s, t \in[0, \infty)$, temos

$$
(t+s)^{p} \geq t^{p}+s^{p}+p t^{p-1} s,
$$

e a estimativa (16) e por fim as Imersões de Sobolev, obtemos que 


$$
\begin{aligned}
I(u) & =\frac{1}{b p^{2}}\left(a+b\|u\|^{p}\right)^{p}-\frac{1}{q+1} \int_{\Omega} h(x)|u|^{q+1} d x-\int_{\Omega} F\left(x, u_{+}\right) d x \\
& \geq \frac{a^{p}}{b p^{2}}+\frac{b^{p-1}}{p^{2}}\|u\|^{p^{2}}+\frac{a^{p-1}}{p}\|u\|^{p}-\frac{\|h\|_{\infty}}{q+1}\|u\|_{q+1}^{q+1}-(\alpha+\varepsilon) \frac{a^{p-1}}{p} \lambda_{1}\|u\|_{p}^{p}-\frac{C_{\varepsilon}}{p^{2}}\|u\|_{p^{2}}^{p^{2}} \\
& \geq I(0)+(1-\alpha-\varepsilon) \frac{a^{p-1}}{p}\|u\|^{p}-\frac{C_{\varepsilon}}{\mu_{1} p^{2}}\|u\|^{p^{2}}-\frac{\|h\|_{\infty} C_{q}}{q+1}\|u\|^{q+1},
\end{aligned}
$$

onde $C_{q}$ é uma constante positiva de modo que

$$
\|u\|_{q+1}^{q+1} \leq C_{q}\|u\|^{q+1}
$$

Devido a condição $(f 2)$, como $\alpha<1$, podemos escolher $\varepsilon>0$ tal que $C_{1}=(1-\alpha-\varepsilon) \frac{a^{p-1}}{p}>0$. Sejam ainda $C_{2}=\frac{C_{q}}{q+1}>0$ e $C_{3}=\frac{C_{\varepsilon}}{\mu_{1} p^{2}}>0$.

Consideremos para cada $t \geq 0$ a função

$$
g(t)=C_{2}\|h\|_{\infty} t^{q+1-p}+C_{3} t^{p^{2}-p} .
$$

Como $0<q<p-1$, temos que $g$ possui mínimo global no ponto

$$
r=C_{4}\|h\|_{\infty}^{\frac{1}{p^{2}-q-1}},
$$

onde $C_{4}=\left(\frac{C_{2}(p-q-1)}{C_{3}\left(p^{2}-p\right)}\right)^{\frac{1}{p^{2}-q-1}}>0$ e então

$$
g(r)=\left(C_{2} C_{4}^{q+1-p}+C_{3} C_{4}^{p^{2}-p}\right)\|h\|_{\infty}^{\frac{p^{2}-p}{p^{2}-q-1}} .
$$

Assim, existe uma constante $\Lambda=\Lambda(a, \alpha, q, p, f, N, \Omega)>0$ tal que se $\|h\|_{\infty}<\Lambda$ então $g(r)<C_{1}$. Portanto, se $\|h\|_{\infty}<\Lambda$, segue pela desigualdade (17) que para todo $u \in W_{0}^{1, p}(\Omega) \operatorname{com}\|u\|=r$ e $\gamma=\left(C_{1}-g(r)\right) r^{p}>0$ que

$$
I(u) \geq I(0)+\gamma .
$$

Resta justificar que existe $v_{0} \in W_{0}^{1, p}(\Omega)$ de modo que $\left\|v_{0}\right\|>r$, tal que $I\left(v_{0}\right)<I(0)$. Para tanto, pelo fato de $\beta>1$ existe $\varepsilon>0$ de modo que $\mu_{1}<(\beta-2 \varepsilon) \mu_{1}$. Assim pela definição de $\mu_{1}$, existe $0 \lesseqgtr v \in W_{0}^{1, p}(\Omega)$ de modo que

$$
\|v\|^{p^{2}}<(\beta-2 \varepsilon) \mu_{1}\|v\|_{p^{2}}^{p^{2}} .
$$

Assim, pela desigualdade (16), segue que

$$
\begin{aligned}
I(t v) & =\frac{1}{b p^{2}}\left(a+b t^{p}\|v\|^{p}\right)^{p}-\frac{t^{q+1}}{q+1} \int_{\Omega} h(x) v^{q+1} d x-\int_{\Omega} F(x, t v) d x \\
& \leq \frac{1}{b p^{2}}\left(a+b\|v\|^{p}\right)^{p}-t^{q+1} \frac{\|h\|_{\infty}}{q+1}\|v\|_{q+1}^{q+1} d x+t^{p} \frac{C_{\varepsilon}}{p}\|v\|_{p}^{p}-t^{p^{2}} \frac{1}{p^{2}}(\beta-\varepsilon)\left[b^{p-1} \mu_{1}\|v\|_{p^{2}}^{p^{2}}\right] \\
& \leq \frac{1}{p^{2}}\left(a b^{-\frac{1}{p}}+\left[b^{p-1}(\beta-2 \varepsilon) \mu_{1} t^{p^{2}}\|v\|_{p^{2}}^{p^{2}}\right]^{\frac{1}{p}}\right)^{p}-\frac{t^{q+1}}{q+1} \int_{\Omega} h(x) v^{q+1} d x-\int_{\Omega} F(x, t v) d x \\
& \leq \frac{1}{p^{2}}\left(a b^{-\frac{1}{p}}+\left[b^{p-1}(\beta-2 \varepsilon) \mu_{1} t^{p^{2}}\|v\|_{p^{2}}^{p^{2}}\right]^{\frac{1}{p}}\right)^{p}-t^{q+1} \frac{\|h\|_{\infty}}{q+1}\|v\|_{q+1}^{q+1}+t^{p} \frac{C_{\varepsilon}}{p}\|v\|_{p}^{p}-t^{p^{2}} \frac{1}{p^{2}}(\beta-\varepsilon)\left[b^{p-1} \mu_{1}\|v\|_{p^{2}}^{p^{2}}\right] .
\end{aligned}
$$

Portanto, pela desigualdade acima e o fato que $p<p^{2}$ e $\beta-2 \varepsilon<\beta-\varepsilon$, segue que

$$
I(t v) \rightarrow-\infty
$$

quando $t \rightarrow \infty$. Logo, existe $t_{0}>0$ suficientemente grande, onde se $v_{0}=t_{0} v$, temos que $v_{0} \in W_{0}^{1, p}(\Omega) \operatorname{com}\left\|v_{0}\right\|>r$ e tal que $I\left(v_{0}\right)<I(0)$. 


\section{Demostração dos resultados}

Procedemos inicialmente a demonstração do Teorema 1.1, que nos garante a existência de uma solução para o problema (8).

Demonstração do Teorema 1.1: Para cada $R>0$ consideramos

$$
B_{R}=\left\{u \in W_{0}^{1, p}(\Omega):\|u\| \leq R\right\} .
$$

Sejam $r>0$ obtido no Lema 2.2. Como $B_{r}$ é um conjunto fechado em $\left(W_{0}^{1, p}(\Omega),\|\cdot\|\right)$, o qual é um espaço de Banach, decorre que $\left(B_{r},\|\cdot\|\right)$ é também um espaço métrico completo. Novamente, pelo Lema 2.2, temos existe $\gamma>0$ de modo que

$$
I(u) \geq I(0)+\gamma, \forall u \in \partial B_{r}
$$

Ainda, note que por $I$ ser um funcional contínuo em $B_{r}$, está bem definido o valor

$$
c_{1}=\inf \left\{I(u): u \in B_{r}\right\}
$$

Observermos que pela definição de $c_{1}$ e a estimativa acima temos

$$
c_{1} \leq I(0)<I(0)+\gamma \leq \inf _{u \in \partial B_{r}} \phi(u)
$$

Dado $h \in L^{\infty}(\Omega)$, consideremos $v \in C_{c}^{\infty}\left(\Omega_{0}\right)$, onde $v \not \leq 0 \mathrm{e}$

$$
\int_{\Omega} h(x) v^{q+1} d x>0 .
$$

Então, pela relação (16), considerando $\varepsilon>0$ de modo que $\beta-\varepsilon>0$, segue que

$$
\begin{aligned}
I(t v) & =\frac{1}{b p^{2}}\left(a+b t^{p}\|v\|^{p}\right)^{p}-\frac{t^{q+1}}{q+1} \int_{\Omega} h(x) v^{q+1} d x-\int_{\Omega} F(x, t v) d x \\
& \leq I(0)+\frac{1}{b p^{2}}\left[\left(a+b t^{p}\|v\|^{p}\right)^{p}-a^{p}\right]-C t^{q+1}+\frac{t^{p} C_{\varepsilon}}{p}\|u\|_{p}^{p} .
\end{aligned}
$$

Logo, para $t \rightarrow 0^{+}$como $q+1<p$, obtemos $I(t v)<I(0)$. Mas para $t>0$ de modo que $t\|v\|<r$, temos que $t v \in B_{r}$ o que implica pela definição de $c_{1}$ que

$$
c_{1} \leq I(t v)<I(0) .
$$

Portanto, pela relação $(18)$ e pelo Princípio Variacional de Ekeland, temos que existe $\left(u_{n}\right) \subset B_{r}$ sequência $(P S)_{\mathcal{C}_{1}}$ para o funcional $I$. Agora pelo fato que $\left(u_{n}\right)$ ser limitada em $W_{0}^{1, p}(\Omega)$, pela Observação 2.1 podemos supor que $0 \leq u_{n}$. Em virtude do Lema 2.1 existem $0 \leq u_{1} \in W_{0}^{1, p}(\Omega)$ tal que, a menos de subsequência, $u_{n}$ converge fortemente para $u_{1}$ em $W_{0}^{1, p}(\Omega)$.

Assim, se $1<q+1<p$, pela continuidade de $I$, obtemos $I\left(u_{1}\right)=c_{1}<I(0)$, donde segue que $u_{1} \geqslant 0$ e pela continuidade da derivada $I^{\prime}$ temos $I^{\prime}\left(u_{1}\right)=0$, isto é, $u_{1}$ é um ponto crítico do funcional $I$ e consequentemente uma solução fraca do problema (8).

Além disso, se considerarmos $h(x) \geq 0$, obtemos $\Delta_{p} u_{1} \leq 0$. Logo, pelo Princípio do Máximo Forte (Vazquez, 1984) existem duas possibilidades, ou $u_{1}>0$ em $\Omega$ ou $u_{1} \equiv 0$ q.t.p. em $\Omega$. Entretanto, como $I\left(u_{1}\right)=c_{1}<I(0)$, segue que $u_{1}>0$ em $\Omega$, o que prova o Teorema 1.1.

Vamos agora, através do Teorema do Passo da Montanha, garantir a existência de uma segunda solução não negativa, do problema (8).

Demonstração do Teorema 1.2: Sejam $r, \gamma, \Lambda>0$ dados no Lema 2.2. Suponhamos que $h$ satisfaz a condição $(h 1)$ e $\|h\|_{\infty}<\Lambda$. Aplicando o Teorema do Passo da Montanha com $X=W_{0}^{1, p}(\Omega)$ e $\phi=I$ temos que para

$$
c=\inf _{\sigma \in \Gamma} \max _{t \in[0,1]} I(\sigma(t)) \geq I(0)+\gamma
$$


existe uma sequência $\left(u_{n}\right) \subset W_{0}^{1, p}(\Omega)$ tal que $\left(u_{n}\right)$ é uma sequência $(P S)_{c}$ para o funcional $I$.

Devemos mostrar que $\left(u_{n}\right)$ é uma sequência convergente. Para tanto, pelo Lema 2.1, basta mostrar que $\left(u_{n}\right)$ é limitada em $W_{0}^{1, p}(\Omega)$.

Suponhamos que isso não ocorra, isto é, $\left\|u_{n}\right\| \rightarrow \infty$, quando $n \rightarrow \infty$ e seja

$$
w_{n}=\frac{u_{n}}{\left\|u_{n}\right\|}
$$

Claramente, $w_{n}$ é limitada em $W_{0}^{1, p}(\Omega)$, pois $\left\|w_{n}\right\|=1$. Logo podemos supor, a menos de subsequência, que existe $w_{0} \in W_{0}^{1, p}(\Omega)$ de modo que $w_{n} \rightarrow w_{0}$ em $W_{0}^{1, p}(\Omega), w_{n} \rightarrow w_{0}$ em $L^{r}(\Omega)$, se $1 \leq r<p^{*}$ e $w_{n} \rightarrow w_{0}$ q.t.p. em $\Omega$.

Mostremos agora que $w_{0} \equiv 0 \mathrm{em} \Omega$. Caso $w_{0} \not \equiv 0 \mathrm{em} \Omega$, temos que $\int_{\Omega}\left|w_{0}\right| p^{2} d x>0$. Assim, pela condição $(f 2)$ e do fato que $f \in C(\bar{\Omega} \times[0, \infty))$, que existe $C_{\varepsilon}>0$ onde para todo $s \in[0, \infty)$

$$
F(x, s) \geq(1-\varepsilon) \frac{b^{p-1}}{p^{2}} \mu_{1} s^{p^{2}}-C_{\varepsilon} s^{p} .
$$

Então, por $\left(u_{n}\right)$ ser uma sequência $(P S)_{c}$ para o funcional $I$, por $\left\|u_{n}\right\| \rightarrow \infty$ e $q<p^{2}$, temos

$$
\begin{aligned}
o(1) & =\frac{1}{\left\|u_{n}\right\|^{2}}\left(I\left(u_{n}\right)+\frac{1}{q+1} \int_{\Omega} h(x)\left|u_{n}\right|^{q+1} d x\right) \\
& =\frac{1}{b p^{2}}\left(\frac{a}{\left\|u_{n}\right\|^{p}}+b\right)^{p}-\int_{\Omega} \frac{F\left(x, u_{n_{+}}\right)}{\left\|u_{n}\right\|^{p^{2}}} d x
\end{aligned}
$$

donde segue que

$$
\lim _{n \rightarrow \infty} \int_{\Omega} \frac{F\left(x, u_{n_{+}}\right)}{\left\|u_{n}\right\|^{p^{2}}} d x=\frac{b^{p-1}}{p^{2}} \lim _{n \rightarrow \infty} \int_{\Omega} \frac{F\left(x, u_{n_{+}}\right)}{\left\|u_{n}\right\|^{p^{2}}} d x=\frac{b^{p-1}}{p^{2}} .
$$

Então, pela igualdade acima, a relação (19) e por $p^{2}>p$, segue que

$$
\begin{aligned}
\frac{b^{p-1}}{p^{2}} & =\lim _{n \rightarrow \infty} \int_{\Omega} \frac{F\left(x, u_{n_{+}}\right)}{\left\|u_{n}\right\| p^{2}} d x+C_{\varepsilon} \frac{\left\|v_{n}\right\|_{p}^{p}}{\left\|u_{n}\right\|^{p^{2}-p}} \\
& \geq \lim _{n \rightarrow \infty}(1-\varepsilon) \frac{b^{p-1}}{p^{2}} \mu_{1}\left\|w_{n}\right\|_{p^{2}}^{p^{2}} \\
& =\lim _{n \rightarrow \infty}(1-\varepsilon) \frac{b^{p-1}}{p^{2}} \mu_{1}\left\|w_{0}\right\|_{p^{2}}^{p^{2}}
\end{aligned}
$$

Dessa forma, temos que

$$
(1-\varepsilon) \mu_{1}=\frac{1}{\left\|w_{0}\right\|_{p^{2}}^{p^{2}}} \geq \frac{\left\|w_{0}\right\|^{p^{2}}}{\left\|w_{0}\right\|_{p^{2}}^{p^{2}}} \geq \mu_{1},
$$

o que é uma contradição pois $\varepsilon>0$. Portanto $w_{0} \equiv 0$ em $\Omega$.

Segue de $(f 3)$ e do fato que $f \in C(\bar{\Omega} \times[0, \infty))$, que existe $C>0$ onde para todo $s \in[0, \infty)$

$$
p^{2} F(x, s)-f(x, s) s \leq C s^{p} .
$$

Assim, como $\left(u_{n}\right)$ é uma sequência $(P S)_{c}$ ilimitada para o funcional $I$, pela relação $(20)$ e $q+1<p$, segue que

$$
\begin{aligned}
o(1)= & \frac{1}{\left\|u_{n}\right\|^{p}} p^{2} I\left(u_{n}\right)-\left\langle I^{\prime}\left(u_{n}\right), u_{n}\right\rangle \\
= & \frac{1}{\left\|u_{n}\right\|^{p}}\left[\frac{1}{b}\left(a+b\left\|u_{n}\right\|^{p}\right)^{p}-\frac{p^{2}}{q+1} \int_{\Omega} h(x)\left|u_{n}\right|^{q+1} d x-\int_{\Omega} p^{2} F\left(x, u_{n_{+}}\right) d x-\left(a+b\left\|u_{n}\right\|^{p}\right)^{p-1}\left\|u_{n}\right\|^{p}\right. \\
& \left.-\int_{\Omega} h(x)\left|u_{n}\right|^{q+1} d x-\int_{\Omega} f\left(x, u_{n_{+}}\right) u_{n_{+}} d x\right] \\
= & \frac{1}{\left\|u_{n}\right\|^{p}}\left[\frac{a}{b}\left(a+b\left\|u_{n}\right\|^{p}\right)^{p-1}+o(1)-\int_{\Omega} p^{2} F\left(x, u_{n_{+}}\right)-f\left(x, u_{n_{+}}\right) u_{n+} d x\right] \\
\geq & \frac{1}{\left\|u_{n}\right\|^{p}}\left[(p-1) a^{p-1}\left\|u_{n}\right\|^{p}-C\left\|u_{n}\right\|_{p}^{p}\right]+o(1) \\
\geq & (p-1) a^{p-1}+o(1)
\end{aligned}
$$

pois $w_{n} \rightarrow 0$ em $L^{p}(\Omega)$. Entretanto a última desigualdade é uma contradição pois $a>0$ e $p-1>0$. 
Portanto, $\left(u_{n}\right)$ é uma sequência limitada em $W_{0}^{1, p}(\Omega)$. Sendo assim, pela Observação 2.1 podemos supor que $u_{n} \geq 0$ e devido o Lema 2.1 existe $0 \leq U_{2} \in W_{0}^{1, p}(\Omega)$ tal que, a menos de subsequência $u_{n} \rightarrow U_{2}$ em $W_{0}^{1, p}(\Omega)$. Uma vez que o funcional $I \in C^{1}\left(W_{0}^{1, p}(\Omega), \mathbb{R}\right)$, segue que $I^{\prime}\left(U_{2}\right)=0$ e $I\left(U_{2}\right)=c>I(0)>I\left(U_{1}\right)$, onde $U_{1}$ é a solução de (8) obtida no Teorema 1.1. Logo, $U_{2}$ é solução fraca não negativa do problema (8), distinta da solução $U_{1}$.

Caso $h(x) \geq 0$, da mesma forma que na demonstração do Teorema 1.1 obtemos $\Delta_{p} U_{2} \leq 0$ e assim, pelo Princípio do Máximo Forte de (Vazquez, 1984), $U_{2}>0$ q.t.p. em $\Omega$, o que conclui a demonstração.

\section{Referências}

Alves, C., O, Corrêa, F. J. S. A., Figueiredo, G. M. (2010). On a class of nonlocal elliptic problems with critical growth Differential Equations and Applications, 2, 409-417.

Alves, C. O., Corrêa, F. J. S. A., Ma, T. F. (2005). Positive solutions for a quasilinear elliptic equation of Kirchhoff type.Computers and Mathematics with Applications, 49, 85-93.

Cammaroto, F., Vilasi, L. (2011). Multiple solutions for a Kirchhoff-type problem involving the p(x)-Laplacianoperator. Nonlinear Analysis, 74, 1841-1852.

Colasuonno, F., Pucci, P. (2011). Multiplicity of solutions for p(x)-polyharmonic elliptic Kirchhoff equations. NonlinearAnalysis: Theory, Methods \& Applications, 74, 5962-5974.

Corrêa, F., Figueiredo, G. (2006). On an elliptic equation of p-Kirchhoff type via variational methods. Bulletin of theAustralian Mathematical Society, 74, 263-277.

Dai, G. (2009). Infinitely many solutions for a p(x)-Laplacian equation in RN. Nonlinear Analysis: Theory, Methods \&Applications, 71, 1133-1139.

Dai, G., Liu, D. (2009). Infinitely many positive solutions for a p(x)-Kirchhoff-type equation. Journal of MathematicalAnalysis and Applications, 359, 704-710.

Dai, G., Wei, J. (2010). Infinitely many non-negative solutions for a p(x)-Kirchhoff-type problem with Dirichletboundary condition. Nonlinear Analysis: Theory, Methods \& Applications, 73, 3420-3430.

Ferrara, M., Khademloob, S., Heidarkhani, J. A. (2014). Multiplicity results for perturbed fourth-order Kirchhoff type elliptic problems. Applied Mathematics and Computation, 234, 316-325.

de Figueiredo, D. G. (1989). Lectures on the Ekeland Variational Principle with applications and detours. Springer-Verlag.

Guo, Y., Nie, J. (2015). Existence and multiplicity of nontrivial solutions for p-Laplacian Schrodinger-Kirchhoff-type equations. Journal of Mathematical Analysis and Applications, 428, 1054-1069.

Hssini, E. M., Massar, M., Talbi, M., Tsouli, N. (2013). Infinitely many solutions for nonlocal elliptic p-Kirchhoff type equation under Neumann boundary condition. International Journal of Mathematical Analysis, 7, 1011-1022.

Hssini, E. M., Massar, M., Tsouli, N. (2015). Existence and multiplicity of solutions for a p(x)-Kirchhoff type problems. Bol Soc Paran Mat, 33, 201-215.

Huang, J., Chen, C., Xiu, Z. (2013). Existence and multiplicity results for a p-Kirchhoff equation with a concave-convex term. Applied Mathematics Letters, 26, 1070-1075.

Kirchhoff, G. (1883). Mechanik. Teubner.

Li, S., Wu, S., Zhou, H. S. (2002). Solutions to semilinear elliptic problems with combined nonlinearities. Journal of Differential Equations, 185, 200-224.

Liang, Z., Li, F., Shi, J. (2013). Positive solutions to Kirchhoff type equations with nonlinearities having prescribed asymptotic behavior. Annales de I Henri Poincare (C) Nonlinear Analysis, 31, 155-167.

Miotto, M. L. (2010). Multiple solutions for elliptic problem in RN with critical Sobolev exponent and weight function. Communications on Pure and Applied Analysis, 9, 233-248.

Miotto, M. L. (2014). Multiplicidade de soluções para uma classe de problemas quaselineares críticos em RN envolvendo função peso com mudança de sinal. Ciência e Natura, 36, 367-379.

Pei, R. (2012). On a p-Laplacian equation of Kirchhoff-type with a potential asymptotically linear at infinity. Journal of Mathematical Analysis, 6(27), 1347-1353.

Perera, K., Zhang, Z. (2006). Sign changing solutions of Kirchhoff type problems via invariant sets of descent flow. Journal of Mathematical Analysis and Applications, 317, 456-463.

Schechter, M. A. (1991). A variation of the Mountain Pass lemma and applications. Journal London Math Soc, 44, 491-502.

Sun, J. J., Tang, C. L. (2011). Existence and multiplicity of solutions for Kirchhoff type equations. Nonlinear Analysis, 74, 1212-1222.

Vazquez, J. L. (1984). Strong Maximum Principle for some quasilinear elliptic equations. Applied Mathematics and Optimization, 12, 191-202. 